\title{
Zwei Verben des Tötens und die inhärente Perspektivierung von Lexemen
}

\author{
Roland Wagner
}

There are two synonymous verbs of killing in German (töten and umbringen), which, however, slightly differ in their inherent perspectivization. While the first one presents the event exclusively as other-directed situation, the second one is indifferent towards the way the action is directed. As a result, the corresponding nominalization (das Umbringen) can refer to a situation of murder as well as to a situation of suicide. In the first part of the article, evidence from the Mannheim corpus of written language is presented to support these observations. In the second part, the possibilities to integrate these facts into a formal model of language are explored.

verbs of killing - middle - middle verbs - reflexive verbs - perspectivization - functional grammar

Im Deutschen gibt es zwei verschiedene, nahezu synonyme Verben des Tötens: töten und umbringen. Die beiden Verben unterscheiden sich jedoch leicht hinsichtlich der Perspektivierung. Während töten eindeutig eine andersgerichtete Situation bezeichnet, ist umbringen hinsichtlich der Ausrichtung der Handlung neutral. Die Folge ist, dass der nominalisierte Infinitiv das Umbringen sowohl Mordhandlungen als auch Selbstmorde bezeichnen kann. Im ersten Teil des Artikels werden Daten aus dem Mannheimer Korpus der geschriebenen Sprache präsentiert, die geeignet sind, die eben vorgetragene Behauptung zu untermauern. Im zweiten Teil wird nach Wegen gesucht, die Daten in ein Grammatikmodell zu integrieren.

Verben des Tötens - Medium - mediale Verben - Mediumverben - reflexive Verben Perspektivierung - funktionale Grammatik

\section{Das Programm des vorliegenden Artikels}

Die folgenden Überlegungen verstehen sich als ein Beitrag zur Theorie der Perspektivierung von natürlichsprachlichen Zeichen. Dass relationale sprachliche Zeichen inhärent perspektiviertsind, gehörtzu den Grundannahmen der funktionalen Syntax (vgl. z. B. WELKE: 2002, 135). Mit „Perspektiviertheit“ ist zunächst einmal gemeint, dass die an sich ungeordneten Relatpositionen eines logischen Prädikats im Prozess der einzelsprachlichen Lexikalisierung in eine syntaktisch-pragmatische Reihenfolge gebracht werden, was sich z. B. auf die „Aktantisierung“ und die damit verbundene Zuweisung von syntaktischen Positionen auswirkt (HELBIG: 1982, 16). Beim Verb mieten, wie z. B. in Peter hat die Wohnung von Frau Schwarz gemietet, wird eine Situation aus Sicht des Mieters beschrieben, dem vom Verb die Rolle des Erstaktanten zugewiesen wird. Das Verb vermieten, welches im Prinzip dieselbe außersprachliche Situation beschreibt, ist dagegen vom Vermieter aus perspektiviert 
und begründet eine Satzstruktur wie z. B. Frau Schwarz hat die Wohnung an Peter vermietet mit dem Vermieter als Erstaktanten.

Im Folgenden werde ich auf eine Perspektivierung anderer Art eingehen, die zwar subtilere, nichtsdestoweniger aber nachweisbare Spuren im syntaktischen Bau des Deutschen hinterlässt: die Perspektivierung von Verben als selbstgerichteten (intrinsischen) oder andersgerichteten (extrinsischen) Prädikaten.

Der erste Abschnitt stellt einen Unterschied in den interpretatorischen Präferenzen zweier nominalisierter Infinitive (Töten $\times$ Umbringen) vor und dokumentiert entsprechende Korpusdaten aus dem Mannheimer Korpus der geschriebenen Sprache. Im zweiten Abschnitt wird nach einer Erklärung für die beschriebenen Unterschiede gesucht. Die entscheidende Ursache glaube ich dabei in den Selektionseigenschaften der beiden Basisverben gefunden zu haben (Abschnitt 3). Im folgenden Abschnitt mache ich mir Gedanken über eine mögliche linguistische Modellierung der vorgestellten Daten, wobei die Theorie der medialen Verben (KEMMER: 1994; KAUFMANN: 2003; 2007; ÁGEL: 2000; 2004) herangezogen wird. Eine kurze Schlussbemerkung weist dann auf die theoretischen Kosten der gewählten Lösung hin.

\section{Zwei Verben des Tötens im Deutschen}

Manche Sprecher des Deutschen, darunter auch der Autor dieses Artikels, ${ }^{1}$ präferieren bei folgenden zwei Sätzen, die - in nominalisierter Form - zwei im Prinzip synonyme Verben enthalten, jeweils unterschiedliche Interpretationen. Die Sätze lauten:

(1) Zum Umbringen ist später noch Zeit.

(2) Zum Töten ist später noch Zeit.

Das Verb umbringen wird in DUDENs Bedeutungswörterbuch $(1985,665)$ als ,gewaltsam ums Leben bringen' definiert, töten als ,den Tod eines Lebewesens herbeiführen, verschulden' (S. 641). Praktisch identisch lauten die entsprechenden Definitionen im Deutschen Universalwörterbuch (DUDEN: 2001, 1588 f. bzw.

1 Eine systematische Befragung von Sprechern des Deutschen habe ich nicht durchgeführt. Bei sporadischen Nachfragen schlossen sich 3 Sprecher meinem Urteil an, 2 lehnten es ab. Die 3 Sprecher, die mit mir übereinstimmen, stammen wie auch ich aus dem süddeutschen Sprachraum. Ein anonymer Rezensent merkte außerdem an, dass es sich bei der interpretatorischen Präferenz von (1) als intrinsisch um eine dialektale Besonderheit handeln könnte. In der Tat ist auffällig, dass auch viele der Belegstellen aus dem Mannheimer Korpus mit Umbringen aus bayerischen oder österreichischen Zeitungen stammen. Das Material ist aber viel zu spärlich und unsystematisch untersucht, als dass ich hier zu Spekulationen Anlass geben möchte. Allenfalls ließe sich annehmen, das umbringen in substantivierter Form generell häufiger im süddeutschen Sprachraum gebraucht wird; die Annahme, dass der interpretatorische Mechanismus selbst im Norden und Süden verschieden funktioniert, halte ich für wenig wahrscheinlich. 
1635) und dem Großen Wörterbuch der deutsche Sprache (DUDEN: 1999, 3931 bzw. 4054). Im Lexikonartikel von umbringen wird töten dabei jeweils explizit als Synonym angeführt.

In den beiden identischen Beispielsätzen (1) und (2) werden diese beiden Verben in der Form von nominalisierten Infinitiven verwendet, wobei die Nominalisierung deutlich am klitischen Artikel $-m$ kenntlich gemacht ist. Inhaltlich handelt es sich bei beiden Sätzen um neutrale Minimalkontexte, die kaum pragmatische Interpretationshilfen liefern.

Gemäß der nahezu identischen lexikographischen Definition beider Verben (einmal ,gewaltsam ums Leben bringen', einmal ,den Tod herbeiführen') sollte man vermuten, dass (1) und (2) völlig gleich interpretiert werden. Dem ist, zumindest bei dem oben erwähnten Kreis von Sprechern, nicht so. Während (1) in Bezug auf die Gerichtetheit der Verbalhandlung - vom Handelnden weg oder rückbezüglich auf ihn selbst hin gerichtet - zumindest neutral ist und demnach, wenn nicht bevorzugt, so doch zumindest auch an einen Selbstmord denken lässt, ist die Handlung in (2) ganz klar auf ein Opfer hin gerichtet, das nicht identisch mit dem Täter ist. Satz (1) wird mit einer verbalen Struktur der Form jemanden oder sich selbst ums Leben bringen assoziiert, (2) dagegen ausschließlich mit jemanden (anderen) ums Leben bringen.

Da Sprecherurteile bekanntermaßen notorisch unzuverlässig sind (vgl. STORRER: 1992, 89) und darüber hinaus, wie bereits oben durch die Formulierung „manche Sprecher" angedeutet, im vorliegenden Fall nicht alle Sprecher des Deutschen meine Meinung teilen, schien es angebracht, den Befund anhand von Korpusbelegen zu überprüfen.

Im Korpus der geschriebenen Sprache des Instituts für deutsche Sprache in Mannheim ${ }^{1}$ finden sich ca. 1520 Kontexte, die eine Infinitivnominalisierung von umbringen, und ca. 3840 Kontexte, die eine Infinitivnominalisierung von töten enthalten. Beide Formen sind leicht über die Groß- und Kleinschreibung zu identifizieren, die wenigen Fehltreffer (z. B. verbales töten am Satzanfang) lassen sich leicht aussortieren. ${ }^{2}$ Betrachten wir nun zunächst die Belegstellen mit Umbringen.

Sollte der Eindruck, die nominalisierte Form von umbringen verweise gleichermaßen auf einen Mord wie auf einen Selbstmord, eine reale Grundlage haben, wäre zu erwarten, dass das Korpus etwa gleich viele Belege von (das) Umbringen in extrinsischer und intrinsischer Bedeutung enthält. Diese Erwartung ließ sich bei Durchsicht der Belegstellen zunächst nicht bestätigen. Unter den 1520 Treffern konnten nur 5 als eindeutig selbstgerichtet bestimmt werden. Eines dieser

1 Das Korpus ist über den Korpus-Manager COSMAS II online zugänglich, vgl. http://www.idsmannheim.de/cosmas2/.

2 Die Fehltreffer, die sich im einstelligen Bereich bewegen, haben mich oben veranlasst, nur ungefähre Angaben zur Zahl der Belegstellen zu machen. Eine genauere Bezifferung ist wohl unzweckmäßig und statistisch irrelevant. 
5 Beispiele führe ich unten als (3) an: ${ }^{1}$

(3) Einmal hat eine Frau angerufen, die sich erschießen wollte [...] Und ich hab“ dauernd versucht, ihr beizubringen, daß Umbringen wirklich die schlechteste Lösung ist. Immerhin ist es mir gelungen, sie etwas zu beruhigen.

Bei einer kleineren Zahl von Beispielen ist es nicht möglich, nur mit Hilfe des im Korpus repräsentierten Kontextes eine eindeutig Zuordnung vorzunehmen, bei weiteren ist die Perspektivierung inhärent offen. Unter (4) führe ich ein solches „offenes“ Beispiel an; hier dient die Unbestimmtheit der nominalisierten Form von umbringen dem Autor (Peter Bichsel) offenbar sogar als Ansatzpunkt für einen literarischen Kunstgriff:

(4) [...] und der Weinende spricht von Umbringen, nur von Umbringen, wobei ihm selbst nicht klar ist, ob er davon spricht, daß man ihn umbringen wolle, oder ob er sich selbst umbringen oder ob er jemanden umbringen wolle.

Diesen wenigen Beispielen, die entweder eindeutig selbstgerichtete UmbringHandlungen bezeichnen oder aber zumindest nicht auf eine fremdgerichtete Handlung hin festgelegt sind, steht im Korpus eine unübersehbare Menge von Belegstellen gegenüber, in denen Umbringen eindeutig auf eine andere Person als den Handelnden ausgerichtet ist. Eines fällt jedoch schon bei einer oberflächlichen Durchsicht ins Auge: Die Erdrückende Mehrheit der Belegsätze ist um das Verb (be)drohen herum konstruiert. Zwei typische Beispiele seien hier als (5) und (6) wiedergegeben:

(5) Am Samstag drohte ihr der Rabiate sogar mit dem Umbringen.

(6) Mit dem Umbringen bedrohte am Samstag abend in Innsbruck ein 36jähriger Mann seine Lebensgefährtin, nachdem er sie geschlagen hatte.

Schätzungsweise $95 \%$ der Treffer entfallen auf solche Konstruktionen des nominalisierten Infinitivs Umbringen mit einem dieser beiden Verben. Die Ausrichtung der Handlung des nominalisierten Infinitivs auf ein Patiens, dass durch die Verwirklichung dieser Handlung in negativer Weise betroffen wäre, ist bei vorliegender lexikalischer Besetzung der Konstruktion bereits durch die Bedeutung des finiten Verbs (bedrohen) eindeutig festgelegt: „Bedrohen“ kann man immer nur jemand anderen, nie sich selbst. In der Bedeutung der Gesamtkonstruktion macht sich demnach nicht die inhärente Perspektivierung des Verbs im nominalisierten

1 Alle im Weiteren angeführten Beispiele stammen aus dem Mannheimer Korpus. Auf eine genauere Angabe der Quellentexte verzichte ich hier. 
Infinitiv, sondern die Perspektivierung des Matrixverbs geltend. Ich schließe daraus, dass Beispiele wie (5) oder (6) für die Untersuchung der inhärenten Perspektivierung von Umbringen nicht aussagekräftig sind. Die Konstruktion überschreibt hier durch die Erfordernisse des Matrixverbs drohen bzw. bedrohen die Perspektivierung, die ggf. in der Infinitivnominalisierung von umbringen angelegt ist. Umgekehrt ist es gerade die inhärente Offenheit von Umbringen im Hinblick auf eine mögliche Perspektivierung, die sowohl die extrinsische Bedeutung von (5) und (6), als auch die intrinsische Bedeutung von (3) und die perspektivische Vagheit von (4) ermöglicht. Methodologisch ziehe ich aus dem Gesagten den Schluss, dass für einen quantitativen Vergleich der intrinsischen Fälle von Umbringen mit den extrinsischen Fällen desselben solche Kontexte ausgeschlossen werden müssen, die bereits durch die lexikalische Besetzung der Umgebung des nominalisierten Infinitivs (und hier v. a. durch die Besetzung der Position des Matrixverbs) eine bestimmte Lesart erzwingen. Nur solche Kontexte, die prinzipiell beide Perspektivierungen zulassen, sollten für den quantitativen Vergleich herangezogen werden.

Nun ist es leider in vielen Fällen eine recht subjektive Entscheidung, welche Kontexte man als „prinzipiell offen“ betrachten will. Ich habe daher für den folgenden Vergleich lediglich die Belegstellen mit dem Verb (be)drohen ausgesondert, außerdem noch ca. 10 Belege, die nach dem Muster auch vom Umbringen war die Rede konstruiert waren, weil hier in allen Fällen ein direkter Anschluss an Kontexte mit drohen u. ä. gegeben war. Nach Aussonderung dieser ,verfälschenden“ Kontexte erhielt ich folgendes Ergebnis:

Tabelle 1: Treffer mit Umbringen im Mannheimer Korpus

\begin{tabular}{|l|c|c|}
\hline & absolute Trefferzahl & Prozent \\
\hline extrinsisch (,jemanden umbringen') & 50 & $81 \%$ \\
\hline intrinsisch (,sich selbst umbringen') & 5 & $8 \%$ \\
\hline unbestimmt/nicht bestimmbar & 7 & $11 \%$ \\
\hline Summe & 62 & $100 \%$ \\
\hline
\end{tabular}

Die „bereinigte“ Statistik der verschiedenen Lesarten von Umbringen zeigt also, dass immerhin $8 \%$ der Belegstellen Umbringen in intrinsischer Bedeutung (,Sich-selbst-Umbringen') enthält. Rechnet man das Ergebnis allerdings auf die Gesamttreffermenge um, bleiben magere 0,3\% (was statistisch gesehen wahrscheinlich bereits im Bereich der Irrelevanz liegt).

Es mag der Leserin/dem Leser überlassen bleiben, ob sie/er meiner Argumentation hinsichtlich der Notwendigkeit der Vorsortierung der Belegstellen mit Umbringen und der damit verbundenen statistischen Aufwertung der intrinsischen Fälle folgen will oder nicht; das Ergebnis, dass meine Korpusanalyse in Bezug auf die nominalisierte 
Form des anderen Verbs, d. h. von töten, geliefert hat, dürfte dagegen auch ohne weiterführende Überlegungen zur Interpretation der gewonnenen Daten eindeutig sein: Unter den weit über 3000 aufgefundenen Belegstellen mit Töten konnte ich keinen einzigen Kontext identifizieren, in dem das Töten eindeutig intrinsisch (d. h. in der Bedeutung von ,Sich-selbst-Töten') gebraucht worden wäre, und dies bei einer Belegzahl die mehr als doppelt so hoch ist wie diejenige für Umbringen. Die einzigen Beispiele, die dabei gewisse Zweifel hinsichtlich der Perspektivierung offen lassen, sind Texten zur Problematik der Sterbehilfe entnommen. Unter (7) und (8) führe ich zwei der markantesten Zweifelsfälle an:

(7) Die kranken und sterbenden Menschen erinnern uns daran, daß keiner über den Wert oder Unwert des Lebens eines anderen Menschen zu befinden hat, selbst nicht über das eigene. Daher stellt die Entscheidung zum aktiven Töten immer eine Willkür dar, auch wenn man sie als Geste der Solidarität und des Mitleids ausgeben will.

(8) Ein weiteres, heikles ethisches Problem: Sterbehilfe. «Sterben in Würde, aber nicht Töten» ist dabei der Hauptgrundsatz.

Die Entscheidung darüber, ob hier von der Handlung des Arztes und damit von der Tötung eines anderen die Rede ist, oder von der Handlung des Patienten und damit von einer Selbsttötung, ist in solchen Kontexten durch die Vermischung beider Gesichtspunkte in der Sterbehilfe-Diskussion extrem schwierig. Bei der Sterbehilfe handelt der Arzt ja schließlich nur im Auftrag des todkranken Patienten und ist dementsprechend ein Werkzeug der Selbst-Tötung dieses Menschen. Ich denke dennoch, dass Töten auch hier (im Gegensatz zu (3)) nicht einfach ,Selbstmord" bedeuten kann. In Beispiel (7) ist die Klassifizierung des Tötens als „Geste der Solidarität und des Mitleids" ein deutlicher Hinweis darauf, dass trotz der Vorerwähnung des „eigenen Lebens“ eigentlich die Tötungshandlung des Arztes im Vordergrund steht: Selbstmord aus Mitleid für sich selbst wäre ein seltsames Konzept. In Beispiel (8) legt der weitere Kontext, in dem es um politische Entscheidungen geht, ${ }^{1}$ die Dominanz des Tötungs-Gedanken nahe: Politische Entscheidungen regeln im Allgemeinen eher das Verhalten anderen Personen gegenüber, nicht das Verhalten sich selbst gegenüber.

Trotz der nicht ganz eindeutigen Datenlage möchte ich daher folgende Schlussfolgerung ziehen: Die Nominalisierung von umbringen lässt bei Bedarf eine intrinsische, d. h. selbstgerichtete Interpretation zu, die Nominalisierung von töten dagegen nicht. Dieser Umstand schlägt sich in jeweils verschiedenen interpretativen Präferenzen gegenüber Minimalkontexten wie (1) und (2) nieder.

1 Der erste Satz der Passage, der Beispiel (8) entnommen ist, lautet: Sachpolitik ist wichtig. Aber sie muss immer die Menschen im Auge behalten und muss den Menschen dienen. 


\section{Mögliche Ursachen für den Befund}

Die oben angeführten Korpusdaten können zwar meinen Eindruck, (1) und (2) würden abweichende Interpretationen nahelegen, untermauern, nicht aber eigentlich eine Begründung für diesen Umstand liefern. Warum lässt sich (das) Umbringen auch intrinsisch interpretieren, (das) Töten jedoch nicht? Kehren wir für die Beantwortung dieser Frage noch einmal zu den oben angeführten Lexikoneinträgen zurück und prüfen, ob sich in diesen Einträgen Hinweise finden lassen, die auf einen abweichenden Gebrauch der beiden Verben und damit auf mögliche Gründe für den beschriebenen Unterschied hindeuten.

Bedauerlicherweise sind die von mir konsultierten Wörterbücher der DUDENReihe (1985; 1999; 2001), die auf der anderen Seite im deutschen Sprachraum als normativ maßgeblich gelten können, in ihren Definitionen nur wenig standardisiert und explizit (vgl. MELČUK et al.: 1995 zu Forderungen an ein linguistisch fundiertes Wörterbuch). Das auffälligste Element, das die Definitionen der beiden Verben voneinander unterscheidet, ist das Prädikat, gewaltsam ' in der Paraphrase von umbringen. Ich nehme nicht an, dass die Autoren der jeweiligen Wörterbuchartikel damit sagen wollen, dass töten einen gewaltlosen Vorgang bezeichnet. Auf jeden Fall würde ein Unterschied [ \pm gewaltsam] keine Erklärung für den oben beobachteten Unterschied in der Interpretation der beiden Nominalisierungen liefern. Es wäre wohl wenig plausibel zu behaupten, dass gerade die Infinitivnominalisierung des Verbs mit dem Merkmal [+ gewaltsam] - im Unterschied zu derjenigen des Verbs mit dem Merkmal [- gewaltsam] - auch für selbstgerichtete Handlungen verwendet werden kann.

Ein interessanterer Hinweis verbirgt sich allerdings in den grammatischen Angaben, die DUDEN (1985, 641 bzw. 665) zu den beiden Verben macht. Bemerkenswerterweise wird töten ausschließlich als transitives Verb klassifiziert, umbringen dagegen als transitives Verb, das daneben auch reflexiv verwendet wird. Ein solcher Zusatzvermerk fehlt bei der Eintragung für töten. Es ist schwer zu beurteilen, inwieweit es sich hier um einen Zufall, geschuldet den Platzzwängen der Endredaktion, handelt oder ob eine bewusste Entscheidung des Lexikographen vorliegt. In späteren (und umfangreicheren) Werken der Reihe (DUDEN: 1999, 3931; 2001, 1588) ist der Hinweis auf die Existenz von sich töten immerhin plötzlich enthalten. Trotzdem fällt auf, dass in allen drei der genannten Lexika nur bei umbringen Verwendungsbeispiele ${ }^{1}$ mit Reflexivum angeführt werden. Sollte hier eine vage empfundene Affinität von umbringen mit Selbstmord der Grund für die einseitige Auswahl der Beispielsätze gewesen sein?

Im Folgenden möchte ich die These vertreten, dass die Interpretationspräferenzen der nominalisierten Formen auf eine Spezialisierung der entsprechenden verbalen Formen hinsichtlich der Verwendung in reflexiven Konstruktionen zurückgehen:

1 Konkret handelt es sich um den Beispielsatz „er hat sich (selbst) umgebracht“. 
Während töten im Allgemeinen auf transitive, nicht-reflexive Konstruktionen beschränkt ist, wird umbringen - neben der Verwendung in transitiven Kontexten immer dann gebraucht, wenn von Selbstmord die Rede ist. Diese Spezialisierung auf reflexive Kontexte schlägt dann auf die Interpretation des nominalisierten Infinitivs durch: Umbringen „erbt“ die Interpretation der verbalen Konstruktion sich umbringen.

Auch in diesem Punkt sollten wieder Korpusdaten Aufschluss über die empirische Fundiertheit der vorgetragenen Behauptung geben können. Allerdings ist im Falle der verbalen Formen die Treffermenge sowohl für umbringen (24 466 Treffer) als auch für töten (142 192 Treffer) derart groß, dass eine manuelle Auswertung aller Belege über die Kraft eines Einzelnen geht. Ich habe mich daher mit der Durchsicht von je 100 Belegstellen pro Verb begnügt. Das Ergebnis ist Tabelle 2 zu entnehmen:

Tabelle 2: Syntaktischer Kontext der Verbalformen

\begin{tabular}{|l|c|c|}
\hline & umbringen & töten \\
\hline jemanden & 66 & 93 \\
\hline sich gegenseitig & 6 & 0 \\
\hline sich selbst & 7 & 2 \\
\hline sich & 18 & 1 \\
\hline Summe & 97 & 96 \\
\hline
\end{tabular}

Die Umrechnung der Trefferzahlen in Prozentangaben erübrigt sich diesmal, da die absoluten Zahlen bei ca. 100 Gesamtbelegen ${ }^{1}$ bereits von vorne herein ungefähr den Anteilen in Prozent entsprechen. Unterschieden werden vier Kategorien: transitive Kontexte, reziproke Kontexte, reflexive Kontexte mit dem Intensivierer selbst und rein reflexive Kontexte. Der Grund für die Trennung von Kontexten mit sich selbst und Kontexten mit sich ohne selbst wird weiter unten deutlich werden. Entscheidend für die Argumentation ist Zeile 4 mit den Trefferzahlen für rein reflexive Kontexte. Hier unterscheidet sich, wie vermutet, umbringen mit 18 Treffern deutlich von töten mit nur einem Treffer. Dieser eine Treffer (unten als Beispiel 9 angeführt) weist zudem einige Besonderheiten auf:

(9) «Ohne die Freiheit und Möglichkeit, sich das Leben zu nehmen, hätte ich mich schon längst getötet», zitierte Abbas Kiarostami an der Pressekonferenz in Cannes den rumänischen Philosophen E. M. Cioran.

Offenbar handelt es sich um eine Übersetzung eines fremdsprachigen Zitats ins Deutsche. Noch wichtiger ist, dass das Textbeispiel dem literarisch-philosophischen

1 In der Tabelle werden geringfügig weniger als 100 Belegstellen erfasst, da das ursprüngliche Sample einige Fehltreffer enthielt. 
Diskurs zuzurechnen ist und damit (auch wenn es in keiner Weise als deviant zu bezeichnen ist) nicht völlig dem neutralen Sprachgebrauch entspricht. Die Markiertheit des einzigen Belegs (unter insgesamt 96 Belegstellen) mit töten in einer einfachen reflexiven Konstruktion lässt die Dominanz des Konkurrenzverbs umbringen in solchen Konstruktionen noch deutlicher hervortreten.

Gewiss ist die Tendenz angesichts der geringen Zahl der ausgewerteten Belegstellen hier nur andeutungsweise erkennbar und müsste durch eine weitergehende statistische Auswertung des Korpusmaterials erhärtet werden; die Tatsache, dass sich besagte Tendenz aber bereits bei Betrachtung weniger Belegstellen abzeichnet, in Verbindung mit der (wenn auch unsystematischen) Auswahl entsprechender Beispielsätze in den Wörterbüchern der DUDEN-Reihe (und dem in dieselbe Richtung weisenden Sprachgefühl des Autors dieser Zeilen), sollen jedoch an dieser Stelle vorerst genügen, um den Tatbestand als plausibel erscheinen zu lassen: Offenbar wird im heutigen Deutschen das Verb umbringen in reflexiven Konstruktionen eindeutig gegenüber dem quasi-synonymen Verb töten bevorzugt.

\section{Selektionsbeschränkungen von umbringen und töten}

Mit der Feststellung am Ende des vorangegangene Abschnitts haben wir die Beantwortung der Ausgangsfrage (Warum Umbringen $\times$ Töten?) allerdings nur auf die nächsttiefere Ebene verschoben. Das Verbalsubstantiv Umbringen ist offenbar genau deshalb einer intrinsischen Interpretation zugänglich, weil das entsprechende Verb (umbringen) eine gewisse Affinität zu reflexiven Konstruktionen aufweist. Woher rührt aber jene Affinität? Handelt es sich hier um eine zufällige Spezialisierung im Wortfeld der deutschen Verben des Tötens oder lassen sich für diese Spezialisierung bestimmte Ursachen finden?

Mir scheint, dass für die Beantwortung dieser weiterführenden Frage die Selektionsbeschränkungen relevant sind, die umbringen im Vergleich zu seinem Konkurrenten töten der syntaktischen Umgebung auferlegt. Betrachten wir die konstruierten Beispielsätze (10) bis (13):

(10) Sie haben Hans-Martin Schleier umgebracht.

(11) 'Sie hat die Fliege umgebracht.

(12) "Sie haben die Bakterien umgebracht.

(13) *Sie bringen ihre Zeit um.

Die angeführten Bewertungen stammen von mir und deuten an, dass Sätze mit umbringen um so akzeptabler zu sein scheinen, je höher der Zweitaktant (bzw. der durch den Zweitaktanten spezifizierte Partizipant der jeweiligen Situation) auf einer Belebtheitsskala vom Typ (14) steht (aus LEMMENS: 1998, 103, übernommen). 
(14) menschlich > menschenähnlich > tierisch > belebt > physikalisches Objekt $>$ abstrakt

Im Beispiel (10) wird der Zweitaktant durch den Namen eines menschlichen Wesens spezifiziert und erfüllt damit die Selektionsanforderungen des Verbs umbringen in idealer Weise. Je weiter wir die Skala (14) hinabsteigen, desto seltsamer werden die Sätze, bis wir mit (13) (abstrakte Größe) bei einer ungrammatischen Struktur angelangt sind. Dass die Forderung nach möglichst hohem Rang des Zweitaktanten auf der Belebtheitsskala (14) keine generelle Eigenschaft von Verben des Tötens ist, belegen die Beispielsätze (15) und (16):

(15) Sie töten die Bakterien ab.

(16) Sie schlägt ihre Zeit tot.

Nun sind Selektionsrestriktionen keine „harten“ grammatikalischen Regeln, die keinerlei Verstöße zuließen. Eher handelt es sich um Tendenzen, die in geeigneten Kontexten überschrieben werden können (DANEŠ et al.: 1987, 59). Ich denke dennoch, dass im vorliegenden Fall die Tendenz in eine klare Richtung weist, und zwar genau in die Richtung, die ich eben beschrieben habe. Werfen wir zunächst wieder einen Blick in die einschlägigen Lexika des Deutschen. Unter dem Stichwort umbringen führen weder DUDEN (1985) noch LANGENSCHEIDT (1993) oder WAHRING (1994) Beispielsätze an, bei denen ein Ausdruck unterhalb der menschlichen Ebene zur Spezifizierung des Zweitaktanten verwendet würde; selbst Sätze mit tierischen Opfern sind nicht zu finden. Eine Ausnahme stellt lediglich die phraseologische Wendung das Material ist nicht umzubringen ,ist sehr haltbar' dar, die bei DUDEN $(2001,1635)$ verzeichnet ist. Vom phraseologischen Charakter dieser Fügung zeugt die Immunität gegen grammatische Operationen wie z. B. die Unmöglichkeit der Rückführung auf eine aktive Basisstruktur: *Die Arbeiterlder Druck und die Hitze bringen das Material um.

Die Durchsicht der oben gesammelten Korpusbelege weist in eine ähnliche Richtung. Unter den 100 Belegstellen mit umbringen konnten nur drei gefunden werden, die einen nicht-menschlichen Zweitaktanten aufweisen: Zweimal ist von Tieren die Rede, einmal vom Skisport:

(17) «Wenn sie den Skisport hier umbringen wollen, werden wir weggehen», meinte Hodler.

Völlig analog dazu ist die Situation auch bei Beispielen mit der nominalisierten Form. Belegstellen wie (18), wo ein Tier als Zweitaktant vorkommt, sind vereinzelte Ausnahmen: 
(18) Unlängst hat der Oberste Gerichtshof das Schächten, also das grausame Umbringen eines unbetäubten Tieres, als legal eingestuft.

Demgegenüber ist die Besetzung der Zweitaktant-Position bei töten viel bunter. Immer wieder sind in den Korpussätzen Tiere (Hunde, Elefanten, Papageien, aber auch Eidechsen) von der Tötungshandlung betroffen, daneben auch Pflanzen (Tomaten) und zweimal sogar Abstrakta:

(19) Zur Musik von James White tanzte das Duo eine witzige Liebesgeschichte zwischen zwei ungleichen Partnern, die in den Versuchen der gegenseitigen Anpassung sich selbst aufgeben und in ihrer Blindheit für die wahre Persönlichkeit ihre Liebe töten.

Insgesamt konnte ich unter den 100 durchgesehenen Belegstellen 17 Beispiele finden, in denen explizit ${ }^{1}$ ein nicht-menschliches Patiens vorkommt. Auch bei den Nominalisierungen finden sich wieder entsprechende Beispiele; unten folgt ein Fall mit einem Abstraktum, d. h. einer Entität auf der rangniedrigsten Stufe der Belebtheitsskala (14):

(20) Lauterbachs Thema ist das Töten von Emotion.

Bemerkenswert ist ferner, dass sich eine ähnliche Tendenz bei der Besetzung der Erstaktantenstelle bemerkbar macht. Wieder tendiert hier umbringen zu Aktanten, die Partizipanten am äußersten linken Rand der Belebtheitsskala (14) spezifizieren, wohingegen töten merklich gelockerte Selektionsbeschränkungen aufweist. Töten kann im Prinzip alles und jeder: vom sprichwörtlichen Gärtner, der ja bekanntlich immer der Mörder ist, ${ }^{2}$ bis hin zu Zyankali und Verkehrsunfällen. Als Agens von umbringen kommen dagegen im Wesentlichen nur Menschen ${ }^{3}$ in Frage. Diese Selektionseigenschaft des Verbs umbringen zeigt sich besonders deutlich in Passivkonstruktionen ohne overte Realisierung des „chômeures“:

(21) Bei heftigen Unwettern in den Niederlanden sind zwei Menschen getötet und vierzig verletzt worden.

1 Es ist notwendig, hier von „expliziten“ Belegen zu sprechen, da in manchen Fällen kein Zweitaktant realisiert war und demgemäß nichts über die Art der Besetzung dieser Position ausgesagt werden kann.

2 Für Leser, die mit dieser Bemerkung nichts anfangen können, sei erklärend hinzugefügt, dass es sich hier um eine Anspielung auf das zumindest in den 70er Jahren im deutschen Sprachraum äußerst bekannte Lied „Der Mörder ist immer der Gärtner" von Reinhard Mey handelt.

3 Ausnahmen bestätigen wieder die Regel, wie z. B. die Redensart „Was mich nicht umbringt, macht mich hart". 
In (21) ist eindeutig von Unfallopfern die Rede. Würde man das Verb töten in diesem Kontext durch umbringen ersetzen, würde dies die Nachricht grundlegend ändern: Die Rede wäre nun nicht mehr von Opfern besagter Unwetter, sondern von Menschen, die während der Unwetter von Mördern gezielt ums Leben gebracht wurden.

Die oben vorgestellten Beobachtungen stimmen mit der vielfach erhobenen Behauptung überein, dass Verben immer nur ein enges Spektrum aus der Belebtheitsskala ausschneiden. Je höher das Agens auf der Skala liege, desto höher liege auch das Patiens auf dieser Skala. Beide Partizipanten dürfen nicht zu weit auseinander entfernt liegen, um die „Handlungskette“ nicht zu unterbrechen (siehe LEMMENS: 1998, 103 f. und die dort angeführte Literatur).

Halten wir fest: Die beiden Verben töten und umbringen stellen jeweils verschiedene Anforderungen an ihre syntaktische Umgebung: Während umbringen Zweitaktanten selegiert, die auf einer Belebtheitsskala wie (14) die höchsten Rangpositionen einnehmen, ist töten in Bezug auf seinen Zweit- (und auch Erst-)Aktanten nur wenig spezifisch und lässt auch Ausdrücke für niedrigrangige Entitäten (niedere Lebewesen, Dinge, Abstrakta) zu. Was dieser Umstand mit der Perspetivierung der beiden Lexeme zu tun hat und wie sich dieser Zusammenhang in ein linguistisches Modell einfügen ließe, werde ich versuchen, im letzten Abschnitt zu klären.

\section{Möglichkeiten einer linguistischen Modellierung}

\subsection{Selektionsanforderungen und Perspektiviertheit}

Die Reflexivierung eines Verbs stellt in irgendeiner näher zu bestimmenden Weise eine Identität zwischen zwei syntaktischen Positionen her. In Sätzen wie Peter rasiert sich bewirkt das Reflexivum, dass der Ausdruck Peter so behandelt wird, als besetze er gleichzeitig sowohl die interne als auch die externe Valenzposition ${ }^{1}$ von rasieren. Aus der externen Valenzposition fällt ihm die Rolle des Agens zu, aus der internen Valenzpositionen diejenige des Patiens. Gleichzeitig ist bei rasieren diese „Doppelbesetzung" völlig unproblematisch, da Peter als [+hum], [+konkret] etc. die Selektionsanforderungen beider Positionen gleichermaßen erfüllt. Bei einer anderen Gruppe von Verben, die Strukturen wie Die Preise haben sich erhöht bilden, wird der Konflikt zwischen den Anforderungen der externen Valenzposition und dem Ausdruck, der diese Position besetzt, so gelöst, dass nur die semantische Interpretation der internen Valenzposition (Patiens) und die damit verbundenen Selektionsrestriktionen wirksam werden (vgl. MELIS: 1990, 125). Die NP die Preise wird dementsprechend so interpretiert, als besetze sie lediglich die interne Valenzposition von erhöhen.

1 Ich benutze hier die Terminologie der Modifizierten Valenztheorie, wie sie in Karlík (2000) entwickelt wurde. „Externe Valenzposition“ ist ungefähr gleichbedeutend mit dem Subjekt in traditioneller Terminologie, ,interne Valenzposition“ dagegen mit (direktem) Objekt. 
In unserem Zusammenhang ist es nun wichtig zu sehen, dass die Identifizierung der beiden Positionen desto besser gelingt, je ähnlicher sie in Bezug auf ihre Selektionsanforderungen sind. Im Prinzip lässt sich jedes deutsche Verb, das eine interne Valenzposition öffnet, reflexivieren. So würde ich einen Satz wie Peter isst sich auf (parallel zu Peter isst das Schnitzel auf) zwar als semantisch bizarr, nicht aber als ungrammatisch bezeichnen. Die Selektionsanforderungen von essen lassen sich im aktuellen Kontext, wie bereits oben gesagt, überschreiben, und die Verwendung des Intensivierers selbst kann - wenn Missverstände drohen - die vom Sprecher beabsichtigte Identifizierung der beiden syntaktischen Positionen betonen und eine entsprechende Interpretation beim Hörer sicherstellen (Nein, nein, du hast ganz richtig gehört: Peter isst sich selbst auf). Die Reflexivierung von Verben wie rasieren, die an beide syntaktische Positionen identische oder sehr ähnliche Selektionsanforderungen stellen, einerseits, und die Reflexivierung von Verben wie essen, die an diese Positionen sehr verschiedene Selektionsanforderungen stellen, andererseits, hat jeweils zwei völlig verschiedene Auswirkungen. Im ersten Fall bewirkt die Konvergenz der beiden Positionen in Bezug auf den semantischen Typ der zu erwartenden Partizipanten, dass sie sich leicht zu einer gemeinsamen Position verbinden lassen. Reflexivum und Verb bilden dann das, was ÂGEL (2000, 154) ein „konventionalisiertes Syntagma“ nennt: einen einheitlichen Valenzträger mit nurmehr einem Aktanten. Im zweiten Fall sperren sich die beiden Positionen aufgrund der jeweils verschiedenen Selektionsrestriktionen gegen die Gleichsetzung. Die Reflexivierung in der Syntax ist zwar grundsätzlich möglich, kommt aber über den Status einer okkasionellen Bildung nicht hinaus. Entsprechende Kontexte werden weiterhin als Realisierungsstruktur eines zweiaktantigen Valenzträgers aufgefasst.

Die oben vorgetragenen Überlegungen laufen daraufhinaus, dem Verblexem selbst einen Anteil am Zustandekommen einerganz bestimmten strukturellen Konfiguration zuzuschreiben. Identische oder sehr ähnliche Restriktionsbeschränkungen von interner und externer Valenzposition begünstigen die Reflexivierung, wodurch der Eindruck der intrinsischen Perspektiviertheit des jeweiligen Verbs entsteht. Dieser Eindruck, der durch den Sprachgebrauch entsteht, schlägt sich schließlich im Lexikoneintrag nieder: Es entsteht ein intrinsisch perspektiviertes Lexem.

Eine ähnliche Konzeption scheint mir bei KÖNIG/SIEMUND (2000) vorzuliegen, wo zwischen Situationen, die ,anders gerichtet“ sind, und solchen, die „nicht-anders gerichtet“ sind, unterschieden wird (vgl. v. a. S. 60 f.). Auch hier ist es bereits die Verbbedeutung, die darüber entscheidet, ob eine reflexive Konstruktion als Spezialfall einer normalerweise auf einen anderen Situationsbeteiligten ausgerichteten Handlung und damit als transitiv interpretiert wird (und damit in vielen Sprachen auch die Realisierung eines „schweren Markers“ wie tsch. sebe bzw. eines Intensivierers wie sich selbst notwendig wird), oder ob sie als Versprachlichung einer konventionell selbstgerichteten Handlung und prinzipiell intransitiv 
aufgefasst wird (und damit auch mit einem „leichten Marker“ wie tsch. se bzw. ohne Intensivierer-selbst auskommt).

König und Siemund führen den Unterschied gänzlich auf die konventionalisierten Vorstellungen von gewissen Situationstypen zurück, wie sie sich in bestimmten Sprachgemeinschaften herausbilden. In den meisten menschlichen Gemeinschaften können Situationen wie Körperpflege, Verteidigung oder positive (Selbst-) Einschätzungen als prinzipiell selbstgerichtet gelten; prinzipiell andersgerichtet sind dagegen Situationen, in denen Gewaltanwendung, Kommunikation oder negative (Fremd-)Einschätzungen im Spiel sind. Dementsprechenden sind die zugehörigen Verblexeme entweder inhärent intrinsisch oder aber inhärent extrinsisch.

$\mathrm{Zu}$ beiden Punkten möchte ich hier eine leichte Korrektur anbringen. Zwar spielt die konventionelle Einschätzung bestimmter Situationen als selbst- bzw. fremdgerichtet sicher eine Rolle bei der Lexikalisierung der jeweiligen Verben als intrinsisch bzw. extrinsisch; man sollte aber auch die formalen, rein grammatischen Merkmale nicht vernachlässigen. Oben habe ich dafür argumentiert, dass ein solches Merkmal - die Selektionsbeschränkungen von bestimmten Verben - eine entscheidende Rolle bei der sprachlichen Klassifizierung dieser Verben als intrinsisch bzw. extrinsisch spielt. Dieser rein formale Aspekt macht sich besonders dann geltend, wenn (wie bei umbringen und töten) keine wesentlichen lexikalischen Unterschiede vorliegen, d. h. wenn im Prinzip dieselbe Situation benannt wird und von daher zunächst kein Unterschied in der Perspektivierung zu erwarten wäre.

Die zweite Anmerkung betrifft die grundsätzliche Klassifizierung von Situationen mit Gewaltanwendung als andersgerichtet. König und Siemund folgen hier einer Tradition, die Verben für entsprechende Handlungen als prototypisch transitiv beurteilt. Dieses generelle Bild muss verfeinert werden. Hier sei nur kurz auf LEMMENS $(1998,58)$ hingewiesen, wo unter Verwendung von grammatisch relevanten Kriterien eine detaillierte Binnendifferenzierung im Wortfeld der Verben des Tötens vorgenommen wird. Dem würde ich hinzufügen: Verben des Tötens müssen keineswegs zwangsläufig andersgerichtete (extrinsische) Situationen bezeichnen; es gibt auch Verben des Tötens, die konventionell selbstgerichtete (intrinsische) Situationen bezeichnen. Den konzeptuellen Hintergrund für diese Behauptung bildet die Beobachtung, dass selbstgerichtete, aggressive Handlungen wie Selbstmord, Selbsthass, Selbstkasteiung etc. in vielen Gesellschaften, teils sogar in konventionalisierter Form (man denke an das Harakiri in Japan) vorkommen, sodass erwartet werden kann, dass die Sprache für diese Handlungen auch konventionalisierte Benennungseinheiten zur Verfügung stellt.

Mir scheint, dass der Gegensatz zwischen töten und umbringen einen Meilenstein auf dem Weg hin zur Etablierung einer derartigen Benennungseinheit darstellt. Dabei ist es kein Zufall, dass sich gerade umbringen auf diesen Weg begeben hat. Am Anfang stand wohl eine zufällige Spezialisierung der internen Valenzposition dieses Verbs auf Ausdrücke, die den höchsten Rang auf der Belebtheitsskala einnehmen. 
Es ist möglich, dass diese Spezialisierung der Etymologie des Wortes geschuldet ist, die heute noch durchsichtig ist und auf die Wendungen um etwas bringen verweist (KLUGE: 2002, 940). Dem Basisverb bringen könnte man dabei eine klare Affinität zu Partizipanten auf den oberen Rängen der Belebtheitsskala unterstellen.

Die Spezialisierung der internen Valenzpositionen von umbringen auf vorwiegend menschliche Partizipanten leistet nun der Reflexivierung Vorschub. Bei identischen Selektionsbeschränkungen der internen und externen Position ergibt sich die völlige Identifizierung beider Positionen und die Verschmelzung der beiden Aktanten zu einem einzigen, hybriden Aktanten viel leichter als bei einem Verb wie töten, das an die interne Position keine besonderen Ansprüche stellt und damit in Strukturen verwendbar ist, in denen die semantischen Merkmale der Ausdrücke, die den Erst- und Zweitaktant spezifizieren, miteinander unvereinbar sind. Ich denke, dass genau mit diesem Umstand der extrinsische Charakter von töten einerseits, und die perspektivische Offenheit von umbringen andererseits zusammenhängt.

Bleibt die Frage, wie dieser Unterschied grammatisch zu modellieren wäre. Da die Opposition intrinsisch $\times$ extrinsisch offenbar Folgen für das Sprachsystem hat (vgl. den Unterschied zwischen (1) und (2) am Anfang des Artikels, die Bevorzugte Verwendung von sich vs. sich selbst sowie einige weitere Auswirkungen, die weiter unten beschrieben werden), müssen entsprechende Informationen an irgendeiner Stelle in der Grammatik verankert sein. Eine Möglichkeit einer solchen Modellierung könnte sein, für das deutsche Verbalsystem die Kategorie „Medium“ zu etablieren. Unten betrachte ich kurz zwei dementsprechende Vorschläge aus der neueren Diskussion.

\subsection{Das Medium im Deutschen}

Das Medium ist ursprünglich eine morphologische Kategorie im Verbalsystem der klassischen Sprachen Griechisch und Sanskrit. Die semantischen Gemeinsamkeiten der Mediumformen untereinander sowie deren auffällige semantische Ähnlichkeit mit den reflexiven Verben der modernen indogermanischen Sprachen haben Anlass zu dem Versuch gegeben, das Medium als konzeptuelle Kategorie zu definieren. Nach KEMMER (1994) kodieren mediale Verbformen Situationstypen mit schwacher interner Differenzierung. Auch wenn vom logischen Standpunkt aus auch im medialen Situationstyp zwei semantische Rollen zu unterscheiden sind, werden beide via sprachliche Konzeptualisierung zu einer einheitlichen Partizipantenrolle integriert Damit unterscheidet sich dieser Situationstyp von reflexiven Situationen, in denen trotz referentieller Gleichsetzung der Partizipanten die konzeptuelle Differenzierung der in der Situation implizierten Rollen aufrecht erhalten wird:

In reflexive events, the Initiator acts on itself as it would act on another entity; the reflexive marker is there simply to signal the unusual fact that the different 
participant roles happen to be filled by the same entity. The middle marker, on the other hand, has the basic function of indicating that the two semantic roles of Initiator and Endpoint refer to a single holistic entity without conceptually distinguished aspects. (S. 207)

Obwohl das Deutsche über keine spezielle morphologische Markierung für mediale Bedeutungen verfügt, sind in mehreren Arbeiten Belege dafür angeführt worden, dass die Kategorie „Medium“ auch hier grammatisch relevant ist. ÁGEL (2004, 23-25) versucht zu zeigen, dass der Koordinationstest, der üblicherweise angewendet wird, um Reflexiva in Objektfunktion zu identifizieren (siehe z. B. HELBIG/BUSCHA: 1999, 210), bei genauerem Hinsehen ein zwiespältiges Ergebnis liefert. Wie die Beispiele (22) und (23) zeigen, ${ }^{1}$ führt die Koordination von Reflexivum und NP nur bei starker Betonung des Reflexivums (im Beispiel durch Unterstreichung notiert) zu einem grammatischen Satz; wird die neutrale Deakzentuierung des Reflexivums beibehalten, entstehen ungrammatische Strukturen:

(22) Peter wäscht sich und das Kind.

(23) *Peter wäscht sich und das Kind.

Ágel schließt daraus, dass Beispiel (23) entgegen der Annahme von Helbig und Buscha kein transitives Verb mit einem direkten Objekt, sondern ein mediales Verb mit Medium-Marker enthält. Dementsprechend spaltet er (ÁGEL: 2000, 156) die Klasse der „reflexiven Konstruktionen“ bei HELBIG/BUSCHA (ebd.) in „reflexive Konstruktionen“ (z. B. sich beobachten) und "agentive Mittelverben“ (z. B. sich waschen) auf. Die erste Ágelsche Subklasse würde den extrinsischen Verben entsprechen, die zweite den intrinsischen Verben.

Eine detailliert ausgearbeitete Theorie des Mediums stellt Ingrid Kaufmann in verschiedenen Arbeiten (z. B. KAUFMANN: 2003; 2007) vor. Die Grundannahme dieser Theorie besteht darin, dass in Sprachen mit Mediumsystemen von verschiedenen Verballexemen eine mediale Stammform abgeleitet werden kann, in deren Aktantenrahmen nur der Zweitaktant des ursprünglichen Lexems enthalten ist. Die agentive Lesart bestimmter Mediumverben (wie z. B. sich waschen) kommt nach Kaufmann dadurch zustande, dass das Patiens des Basislexems als potentieller Kontrolleur der benannten Situation gekennzeichnet ist. Beim Aufrücken in die höchste Position des reduzierten Aktantenrahmens kann es daher (zusätzlich zur Patiensbedeutung) die mit dieser Position verbundene Interpretation als Kontrolleur der benannten Situation annehmen. An anderer Stelle (WAGNER: in Druck)

1 Die Beispielsätze einschl. Grammatikalitätsurteil stammen aus Ágel (2004, 23). Dort ist allerdings durch einen Druckfehler das Sternchen abhanden gekommen; dass V. Ágel - wie auch der Autor des vorliegenden Artikels - Satz (23) als ungrammatisch betrachtet, geht eindeutig aus dem Text von Ágel (2004) hervor. 
äußere ich die Vermutung, dass es im Deutschen einen Zusammenhang zwischen der Auszeichnung eines Aktanten als potentiellen Kontrolleur der Situation und den Selektionseigenschaften des entsprechenden Verbs gibt. Aktanten, die nur durch Ausdrücke mit extrem hohen Werten für Belebtheit (gemäß Skala 14) spezifiziert werden können, müssen obligatorisch als (ggf. potentielle) Kontrolleure ausgezeichnet werden, da bei entsprechenden Partizipanten immer angenommen werden kann, dass sie die jeweilige Situation kontrollieren können.

An dieser Stelle möchte ich lediglich auf eine Beobachtung aus KAUFMANN (2003; 2007, 196 ff.) hinweisen, die einen weiteren Beleg für den strukturellen Unterschied von Konstruktionen mit medialen Verben und solchen mit transitiven Verben liefert. Kaufmann konnte bei einer korpusbasierten Studie feststellen, dass die Realisierung bzw. Nicht-Realisierung des Reflexivums bei nominalisierten Infinitiven vom Status des betreffenden Verbs abhängt. Bei medialen Verben wie sich waschen unterbleibt die Realisierung gewöhnlich, bei reflexiv gebrauchten transitiven Verben wie beobachten erfolgt sie dagegen regelmäßig. Ich illustriere den Gegensatz mit zwei konstruierten Beispielen, wobei waschen bzw. anziehen medial, braten dagegen transitiv ist:

(24) Das Waschen und Anziehen fällt der alten Frau schwer.

(25) Das stundenlange Sich-in-der-Sonne-Braten ist ungesund.

(26) Das stundenlange In-der-Sonne-Braten ist ungesund.

Bei (24) kommt die semantisch rückbezügliche Interpretation völlig zwanglos auch ohne das Reflexivum zustande. Die alte Frau ist sowohl Agens als auch Patiens der Verbalhandlung. Die Nominalisierung von braten in (25) benötigt dagegen die unterstützende Realisierung des Reflexivums, um dieselbe agentive Interpretation wie die entsprechende verbale Struktur (Man brät sich in der Sonne) zu motivieren. Ohne Reflexivum (vgl. Beispiel 26) wird die Struktur inchoative interpretiert und auf eine nichtagentive, intransitive Basisstruktur (Man brät in der Sonne) bezogen.

Wenn man die Ausführungen von Ágel und Kaufmann auf unser Problemverb umbringen anwendet, dann gelangt man zu folgendem Schema (frei nach KAUFMANN: 2007, 1694): 


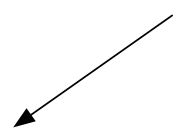

transitiver Stamm:

umbringen

(A-1, A-2)

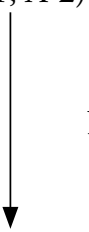

das Umbringen (von Menschen)

(A-1, A-2)
Blockierung von A-1

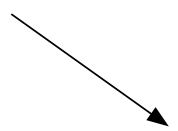

medialer Stamm:

umbringen

(A)

NOMINALISIERUNG

das Umbringen

(A)

Die linke Seite des Schemas zeigt die kanonische Abbildung beider im Basislexem implizierter Aktanen in den Aktantenrahmen des transitiven Stamms. ${ }^{1}$ Beim Einbau in eine verbale Struktur werden beide Aktanten in den dafür vorgesehenen syntaktischen Positionen realisiert: $E_{A-1}$ brachteseine Frau $_{A-2}$ um aber auch: $E_{A-1}$ brachte sich (selbst) und seine Frau ${ }_{A-2}$ um. Wird von diesem Stamm eine Nominalisierung gebildet, bleiben beide Aktanten erhalten und müssen dementsprechend interpretiert werden. Aus Mangel an syntaktischen Positionen können sie jedoch nicht (oder zumindest nicht beide) realisiert werden. Die genaue Analyse hängt an dieser Stelle stark vom gewählten Grammatikmodell ab; der Einfachheit halber stelle ich die Verhältnisse so dar, dass die impliziten Aktanten interpretatorisch mit den Aktanten des Matrixverbs identifiziert werden: $E_{A-1}$ drohte seiner Frau ${ }_{A-2}$ mit dem Umbringen.

Auf der rechten Seite des Schemas ist die reduzierte Abbildung der Aktanten dargestellt, die für die Bildung des medialen Stammes einschlägig ist. Hier wird der höhere der beiden Aktanten unterdrück und nur der tiefere (das Patiens) in den Aktantenrahmen des medialen Verbalstammes abgebildet. Ohne Oppositionspartner unterbleibt dann die Differenzierung nach Erst- und Zweitaktant. Wird dieser Stamm in eine verbale syntaktische Struktur eingebaut, steht nur noch ein Aktant zur Realisierung zur Verfügung: $E r_{A}$ bringt sich um. Dasselbe gilt im Falle, dass der Stamm nominalisiert wird. Da nur ein Aktant im Aktantenrahmen vorhanden ist, steht für die Interpretation nur ein Aktant zur Verfügung; eine semantisch transitive

1 Kaufmann spricht hier von einem ,aktiven“ Stamm. Da Aktiv und Passiv traditionell eher als frei bildbare Formen desselben Verbalstamms (und nicht als Ergebnis von Wortbildungsprozessen) aufgefasst werden, erscheint mir "transitiv“ hier geeigneter. Der Nachteil dieser Terminologie ist allerdings, dass die Parallelität zum Mediumbegriff in den klassischen Sprachen verloren geht, da dort das Medium nicht in Opposition zum Transitivum, sondern zum Aktivum aufgefasst wird. 
Interpretation, die die Existenz von zwei Aktanten voraussetzen würde, ist damit ausgeschlossen, A muss gleichzeitig als Agens und Patiens interpretiert werden: Zum Umbringen hast du später noch Zeit. Aus Gründen der Anschaulichkeit habe ich wieder eine Struktur gewählt, in der der implizierte Aktant mit der Oberflächenrealisierung eines anderen Aktanten interpretatorisch gleichgesetzt wird.

\section{Schlussbemerkung}

Grammatische Modelle werden nicht nur danach beurteilt, wie gut sie die sprachlichen Fakten wiedergegen; ein entscheidendes Kriterium ist immer auch die Darstellungsökonomie. Was den ersten Punkt betrifft, liefert das vorgestellte Modell meiner Meinung nach eine befriedigende Erklärung für den Unterschied zwischen (1) und (2). Es ließe sich sagen, dass von umbringen zwei Stämme gebildet werden können: ein transitiver und ein medialer. Diese Information wäre im Lexikoneintrag von umbringen zu vermerken. Je nachdem, welcher Stamm nun den Ausgangspunkt für die Derivation des nominalisierten Infinitivs bildet, erhalten wir die extrinsische oder die intrinsische Interpretation von (1). Das Verb töten würde dagegen nur ein en, und zwar einen transitiven Stamm bilden (ggf. auch im Lexikon zu vermerken oder aber per Default sicherzustellen). Die intrinsische Interpretation von (2) ist daher nicht möglich; in jedem beliebigen Kontext wären immer zwei Aktanten zu interpretieren. Die Konstruktion mit dem nominalisierten Infinitiv von töten ist daher obligatorisch semantisch transitiv bzw. extrinsisch. In Kontexten wie (9) muss syntaktisch reflexiviert werden, wobei der Zweitaktant durch ein (normalerweise verstärktes) Reflexivpronomen realisiert wird.

Wahrscheinich ist aus diesen Ausführungen auch bereits deutlich geworden, wo bei der vorgestellten Lösung im Hinblick auf Punkt zwei die „theoretischen Kosten“ liegen: In einer Sprache, in der sich kein morphologischer Hinweis auf die Existenz zweier Formen findet, müssten wir annehmen, dass das Reflexivum sich homonym ist. Neben der Existenz eines pronominalen sich, das zur Spezifizierung von Aktanten verwendet werden kann, müssten wir ein weiteres sich postulieren, das nicht als Realisierung eines Aktanten auffassbar wäre und keine andere Funktion hätte, als mediale Verbalstämme zu markieren. Ob diese theoretische Vorschussleistung noch tragbar ist, bleibe an dieser Stelle dahingestellt.

\section{Literatur:}

ÁGEL (2000): Ágel, Vilmos. Valenztheorie. Tübingen: Gunter Narr, 2000.

ÁGEL (2004): Ágel, Vilmos. Prinzipien der Valenztheorie(n), in: Stănescu, Speranța (Hg.):Die Valenztheorie. Bestandaufnahme und Perspektiven. Dokumentation einer wissenschaftlichen Tagung in Sibiu/Hermannstadt im Februar 2002. Frankfurt/Main, Berlin, Bern etc.: Peter Lang, 2004, 11-30.

DANES et al. (1987): Daneš, František/Hlavsa, Zdeněk/Jirsová, Anna/Macháčková, Eva/ Prouzová, Hana/Svozilová, Naděžda. Vétné vzorce v čeśtině. Praha: Academia, 1987. 
DUDEN (1985) Bedeutungswörterbuch. Hrsg. von W. Müller. Mannheim, Wien, Zürich: Duden Verlag, 2. Aufl., 1985.

DUDEN (1999): Duden. Das große Wörterbuch der deutschen Sprache in zehn Bänden. Band 9: Tach - Vida. Hrsg. von Werner Scholze-Stubenrecht/Dieter Mang et al. Mannheim, Leipzig, Wien, Zürich: Duden Verlag, 3. Aufl., 1999.

DUDEN (200I) Deutsches Universalwörterbuch. Mannheim, Leipzig, Wien, Zürich: DudenVerlag, 4. Aufl., 2001.

HELBIG (1982): Helbig, Gerhard. Valenz - Satzglieder - semantischer Kasus - Satzmodelle. Leipzig: VEB Verlag Enzyklopädie, 1982.

HELBIG/BUSCHA (1999): Helbig, Gerhard/Buscha, Joachim. Deutsche Grammatik. Ein Handbuch für den Ausländerunterricht. Leipzig, Berlin, München, Wien, Zürich, New York: Langenscheidt/Verlag Enzyklopädie, 19. Aufl., 1999.

KARLÍK (2000): Karlík, Petr. Hypotéza modifikované valenční teorie, in: Slovo a slovesnost, 2000, 61, Nr. 3, 170-189.

KAUFMANN (2003): Kaufmann, Ingrid. Infinitivnominalisierungen von reflexiven Verben: Evidenz gegen Argumentstrukturvererbung? In: Maienborn, Claudia (Hg.) (A)Symmetrien - (A)Symmetries. Beiträge zu Ehren von Ewald Lang - Papers in Honor of Ewald Lang. Tübingen: Stauffenburg, 2003, 203-232.

KAUFMANN (2007): Kaufmann, Ingrid. Middle voice, in: Lingua, 2007, 117, Nr. 10, $1677-1714$.

KEMMER (1994): Kemmer, Susan. Middle Voice, Transitivity, and the Elaboration of Events, in: Fox, Barbara/Hopper, Paul J. (Hg.): Voice. Form and Function. Amsterdam, Philadelphia: John Benjamins, 1994, 179-230.

KLUGE (2002): Etymologisches Wörterbuch der deutschen Sprache. Hrsg. von E. Seebold. Berlin, New York: Walter de Gruyter, 24. Aufl., 2002.

KÖNIG/SIEMUND (2000): König, Ekkehard/Siemund, Peter. Intensifiers and reflexives: A typological perspective, in: Frajzyngier, Zygmunt/Curl, Traci S. (Hg.): Reflexives. Forms and Functions. Amsterdam, Philadelphia: John Benjamins, 2000, 41-74.

LANGENSCHEIDT (1993): Langenscheidts Großwörterbuch Deutsch als Fremdsprache. Hrsg. von D. Götz, G. Haensch und H. Wellmann. Berlin, München, Wien, Zürich, New York: Langenscheidt, 5. Aufl., 1993.

LEMMENS (1998): Lemmens, Maarten. Lexical perspectives on transitivity and ergativity. Causative constructions in English. (= Amsterdam Studies in the Theory and History of Linguistic Science, Bd. 166). Amsterdam, Philadelphia: John Benjamins, 1998.

MELČUK et al. (1995): Meľcuk, Igor A./Clas, André/Polguère, Alain: Introduction à la lexicologie explicative et combinatoire. Paris: Duculot, 1995.

MELIS (1990): Melis, Ludo. La voie pronominale. La systématique des tours pronominaux en français moderne. Paris: Duculot, 1990.

STORRER (1992): Storrer, Angelika. Verbvalenz. Theoretische und methodische Grundlagen ihrer Beschreibung in Grammatikographie und Lexikographie. Tübingen: Max Niemeyer, 1992.

WAGNER (in Druck): Wagner, Roland. Situation control under reflexivization a comparative study of certain verbs in Czech and German, in: Dočekal, Mojmír/Ziková, Markéta (Hg.): Czech in Formal Grammar. München: Lincom, erscheint voraussichtlich 2009.

WAHRING (1994): Deutsches Wörterbuch. Hrsg. von R. Wahring-Burfeind. Gütersloh: Bertelsmann Lexikon Verlag, 1994.

WELKE (2002): Welke, Klaus. Deutsche Syntax funktional. Perspektiviertheit syntaktischer Strukturen. Tübingen: Stauffenburg, 2002. 